\title{
What is the influence of two strain rates on the relationship between human cortical bone toughness and micro- structure?
}

\author{
Rémy Gauthier ${ }^{1,2}$, Hélène Follet ${ }^{3}$, Max Langer ${ }^{2}$, Françoise \\ Peyrin $^{2,4}$ and David Mitton ${ }^{1, *}$ \\ ${ }^{1}$ Univ Lyon, Université Claude Bernard Lyon 1, IFSTTAR, LBMC UMR_T9406, \\ F69622, Lyon, France ; \\ ${ }^{2}$ Univ Lyon, CNRS UMR 5220, Inserm U1206, INSA Lyon, Université Claude Bernard \\ Lyon 1, Creatis, F69621 Villeurbanne, France ; \\ ${ }^{3}$ Univ Lyon, Université Claude Bernard Lyon 1, INSERM, LYOS UMR1033, F69008, \\ Lyon, France ;
}

${ }^{4}$ ESRF, 38, Grenoble France.

*david.mitton@ifsttar.fr

Abstract: Cortical bone fracture mechanisms are well studied under quasi-static loading. The influence of strain rate on crack propagation mechanisms needs to be better understood, however. We have previously shown that several aspects of the bone microstructure is involved in crack propagation, such as the complete porosity network, including the Haversian system and the lacunar network, as well as biochemical aspects, such as the maturity of collagen cross-links. The aim of this study is to investigate the influence of strain rate on the toughness of human cortical bone with respect to its microstructure and organic non-collagenous composition. Two strain rates will be considered: quasi-static loading $\left(10^{-4} \mathrm{~s}^{-1}\right.$, a standard condition, and a higher loading rate $\left(10^{-1} \mathrm{~s}^{-1}\right)$, representative of a fall. Cortical bone samples were extracted from 8 female donors (50 y.o. to 91 y.o.). Three- point bending tests were performed until failure. Synchrotron radiation micro-computed tomography imaging was performed to assess bone microstructure including the Haversian system and the lacunar system. Collagen enzymatic cross-link maturation was measured using a high performance liquid chromatography column. Results showed that that under quasi-static loading, the elastic contribution of the fracture process is correlated to both the collagen cross-links maturation and the microstructure, while the plastic contribution is correlated only to the porosity network. Under fall-like loading, bone organization appears to be less linked to crack propagation.

Keyword: Human cortical bone, crack propagation, synchrotron micro-computed tomography, Haversian canals, osteocytes lacunae, collagen cross-links, paired anatomical locations 


\section{Introduction}

From a clinical point of view, the prediction of bone fracture risk remains difficult while more than 9 million fractures occur ever years ${ }^{1-3}$. Clinical gold standard methods are still relying on the assessment of bone mineral density (BMD) using Dual-energy X-ray Absorptiometry (DXA) ${ }^{4}$.

BMD is however not sufficient to accurately predict the fracture risk ${ }^{1,2}$. Even if BMD is a good predictor of bone strength ${ }^{5}$, this single parameter does not describe the overall mechanical response of bone in a fall loading configuration ${ }^{6}$. Other parameters, related to the geometry, the material composition or the architecture of bone, have to be considered to understand the bone fracture process ${ }^{7,8}$.

Toughness appears to be one relevant biomechanical parameter to understand bone fracture mechanisms ${ }^{9}$. During crack propagation, a number of toughening mechanisms occur in order to slow and stop the crack during its propagation and thus increase the energy needed to break the material ${ }^{10-14}$. These mechanisms depend on the structural organization of the bone tissue: micro-crack formation is associated to the Haversian network ${ }^{15}$, cracks are deflected along the long axis of the osteons following the cement lines ${ }^{16,17}$ and interfibrillar sliding might be associated to the maturity of the collagen ${ }^{18,19}$.

These so called toughening mechanisms have been widely studied under standard quasistatic loading, but there are only few data considering higher loading rates ${ }^{20-22}$. This despite fractures mainly occurring after falls from a standing position ${ }^{23}$. We recently showed that cortical bone toughness in different anatomical locations is significantly decreased at a higher strain rate compare to a standard quasi-static loading ${ }^{24}$. In order to better understand the bone fracture process in the case of a fall, the influence of microstructure and biochemical composition on the decrease in toughness should be studied.

The aim of this study is thus to investigate relationships between the bone toughness and its structural and compositional properties both under quasi-static and fall-like loading conditions in order to provide new insight regarding cortical bone crack propagation mechanisms. The current study builds on previous results on human cortical bone toughness ${ }^{24}$, microstructure ${ }^{25}$ and collagen cross-link composition ${ }^{26}$. 


\section{Materials and methods}

\section{Bone samples}

The investigations were performed on 8 paired femoral diaphyses, femoral necks and radial diaphyses extracted from female donors (average: 70 y.o, min: 50 y.o., max: 91 y.o.). No medical history of the donors was available. After extraction, the bones were kept hydrated within gauze soaked with saline and stored at $-20{ }^{\circ} \mathrm{C}$ until sample preparation.

\section{Biomechanical measurements}

The biomechanical experiments were performed following the experiment protocol used in ${ }^{24}$. Two contiguous rectangular specimens from each bone, between 20 and $25 \mathrm{~mm}$ long, $2.1 \pm 0.1 \mathrm{~mm}$ width and $1.0 \pm 0.1 \mathrm{~mm}$ thick, their long axis parallel to the main axis of the bone, were prepared using a low speed saw with a diamond coated blade (ISOMET 4000, Buehler, USA). They were then notched in their middle perpendicularly to the long axis of the diaphysis and subjected to a three point bending test until failure (Figure 1). Each contiguous sample was submitted either to standard quasi-static loading $\left(10^{-4} \mathrm{~s}^{-1}\right)$ or fall-like loading $\left(10^{-1} \mathrm{~s}^{-1},{ }^{27}\right)$ to assess the influence of the loading rate. Samples were rehydrated in saline solution $24 \mathrm{~h}$ before the mechanical tests. Toughness parameters were then investigated using the ASTM E- $1820^{28}$. The details can be found in ${ }^{24}$.

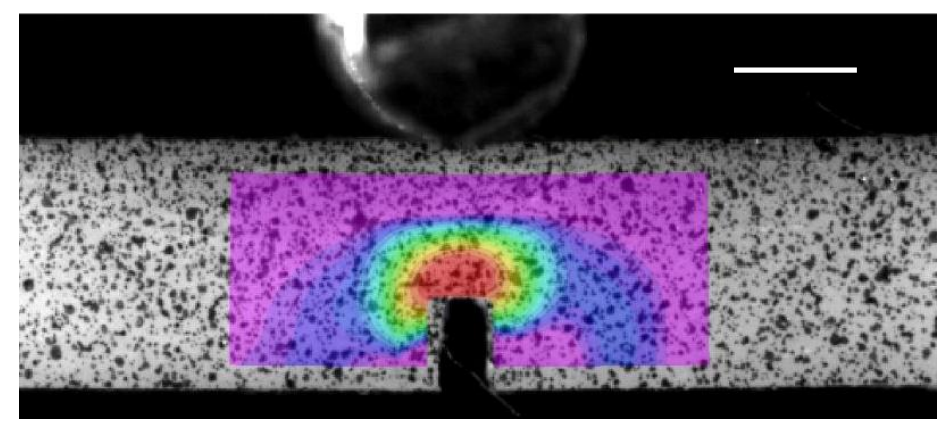

Figure 1 Cortical SENB sample under a critical loading following the ASTEM E-1820. Strain field is calculated using Digital Image Correlation (Vic-2D, Correlated Solutions, Columbia, USA). Red color represents the highest value of strain. Note the strain concentration around the notch. Scale bar: $1 \mathrm{~mm}$.

The parameters assessed in the current study are the elastic contribution to toughness $\mathrm{J}_{\mathrm{el}}$ $\left(\mathrm{kN} \cdot \mathrm{m}^{-1}\right)$, the plastic contribution $\mathrm{J}_{\mathrm{pl}}\left(\mathrm{kN} \cdot \mathrm{m}^{-1}\right)$, and the global toughness parameter $\mathrm{J}=\mathrm{J}_{\mathrm{el}}+$ $\mathrm{J}_{\mathrm{pl}}\left(\mathrm{kN} \cdot \mathrm{m}^{-1}\right)$. They give the energy needed to initiate $\left(\mathrm{J}_{\mathrm{el}}\right)$ and propagate $\left(\mathrm{J}_{\mathrm{pl}}\right)$ a crack through bone structure ${ }^{24}$. These parameters are called the energy release rates, and are closely related to the toughness ${ }^{29}$. For the sake of simplicity, we will use the term toughness when referring to these parameters. 


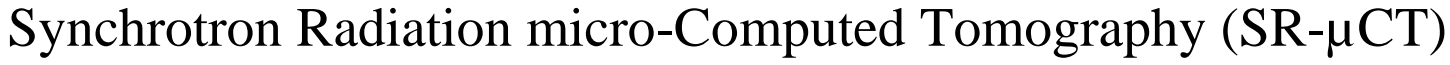

After the biomechanical experiments, Synchrotron Radiation micro-Computed

Tomography (SR- $\mu \mathrm{CT}$ ) imaging was used to assess cortical bone microstructure from the Haversian system down to the lacunar system in the samples ${ }^{25}$. The scanned region of interest was chosen outside the rollers of the mechanical set up so as to not be affected by the mechanical loading. The fractured samples were delipidated one week before imaging: the samples were immerged in acetone for 30 minutes, then rinsed with water, and finally dehydrated by successive immersion in $70 \%$ and nearly $100 \%$ ethanol baths for a maximum period of 2 days. Image acquisition was performed on beamline ID19 at the European Synchrotron Radiation Facility (ESRF, Grenoble, France) using a "pink beam" filtered undulator radiation with an effective energy of $31 \mathrm{keV}$. Volumes of $2048^{3}$ voxels with a nominal voxel size of $0.7 \mu \mathrm{m}$ were finally obtained from each sample.

In the current study, we mainly consider the following structure parameters: the Haversian canal diameter (On.Ca.Dm, in $\mu \mathrm{m}$ ), the lacunar density (Lc.N/BV, in $\mathrm{mm}^{-3}$ ) and the average lacunar radius of curvature (Lc. $\rho_{1}$, in $\left.\mu \mathrm{m}\right)$ defined as:

$L c . \rho_{1}=\frac{\left(L c . L_{2}\right)^{2}}{L c . L_{1}}$

where Lc.L $L_{1}(\mu \mathrm{m})$ and Lc. $L_{2}(\mu \mathrm{m})$ are the dimensions of the first and second axis of an ellipsoid fitted to the lacuna, respectively ${ }^{30}$.

\section{Biochemical measurements}

The collagen cross-links were measured using a high performance liquid chromatography column as done in ${ }^{26}$. These measurements were performed on bone tissue that remained from the preparation of samples for the mechanical tests, i.e. tissue adjacent to these samples in the bone. In the current study, we focused on the CX ratio which is the ratio between divalent (immature) and trivalent (mature) enzymatic cross-links. This parameter reflects the maturation of the collagen matrix: a more mature collagen matrix implies a lower CX ratio.

\section{Statistical tests}

In order to make statistical tests on more than 8 samples, a longitudinal study was performed by pooling the three anatomical locations to finally have a population of 24 samples for each biomechanical, microstructural or biochemical parameter ( 8 femoral diaphyses, 8 femoral necks and 8 radial diaphyses). Analyses were also performed for each individual site to have information on their respective contributions.

Shapiro-Wilk's normality tests were not satisfied for some of the parameters. Therefore, Spearman's rank correlation coefficient was used to investigate correlations between the measured parameters. 
Multiple linear regressions using backward stepwise elimination to remove the nonsignificant parameters were performed until obtaining significant relation to explain the variance of the biomechanical parameters. Statistical significances were considered at $\mathrm{p}<0.05$. The statistical analysis was performed using StatView (StatView, Abaqus, USA).

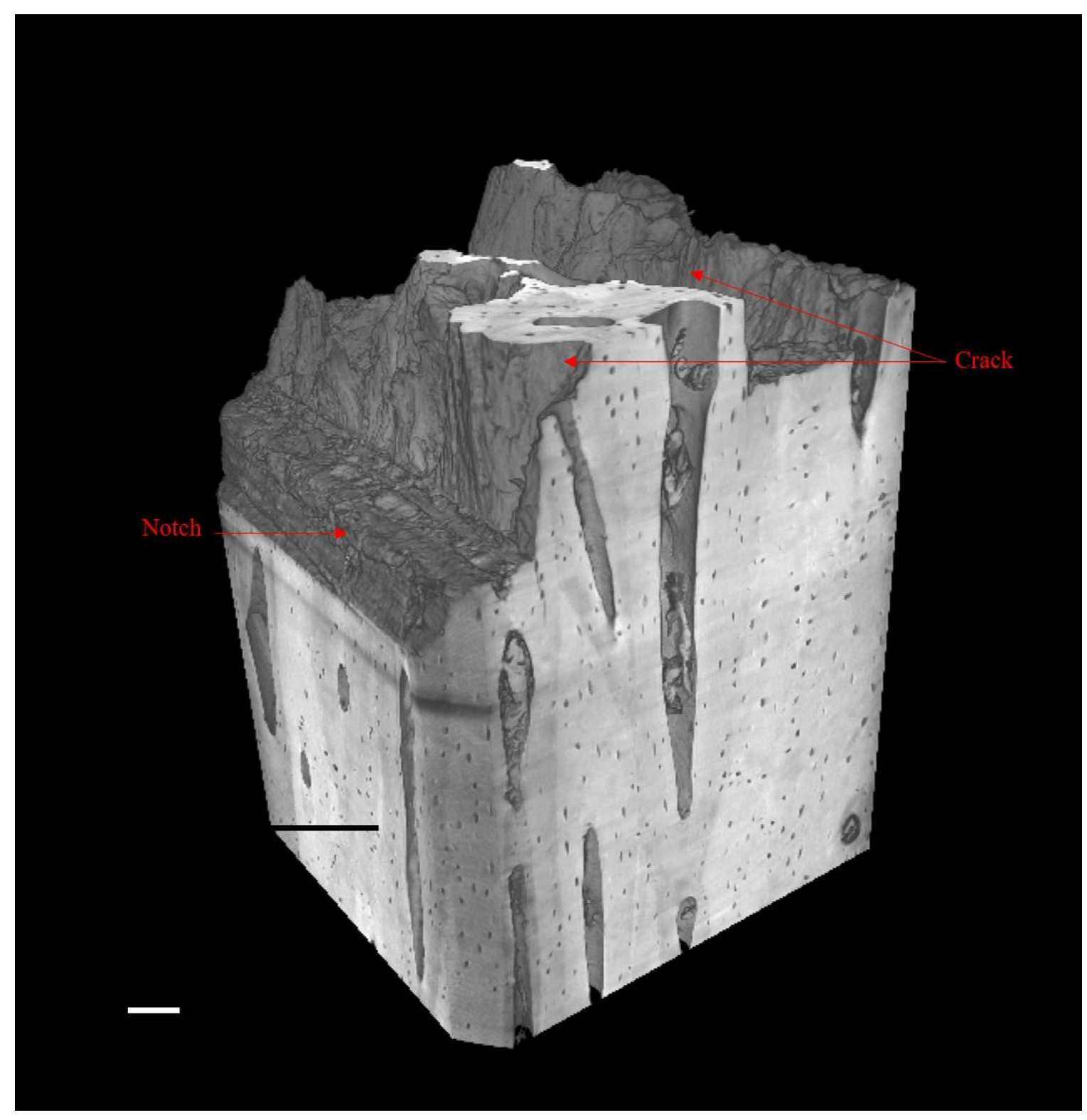

Figure 2 Volume rendering of a radial diaphysis sample after subjected to critical loading (woman, 73 y.o., scale bar $=500 \mu \mathrm{m}$ )

\section{Results}

Figure 2 illustrates a volume rendering of one radius sample imaged after critical loading. On this 3D display, we can observe the Haversian canals appearing as the long 
longitudinal porosities and the osteocyte lacunae as small porosities. Table 1 presents the Spearman's coefficients between biomechanical and structural or biochemical features. The detailed correlation for each anatomical location is shown on the table S1, S2 and S3 of the supplementary materials.

We first consider each location separately (Table S1, S2 and S3 in supplementary materials).

In the femoral diaphysis, under quasi-static loading $\mathrm{J}_{\mathrm{pl}}$ and $\mathrm{J}$ is negatively correlated to lacunar density. Under fall-like loading, $\mathrm{J}_{\mathrm{el}}$ is positively correlated with the $\mathrm{CX}$ ratio, and $\mathrm{J}_{\mathrm{pl}}$ and $\mathrm{J}$ are negatively correlated to Lc.N/BV (Table S1).

In the femoral neck, under quasi-static loading no significant correlations were found. Under fall-like conditions, however, $\mathrm{J}_{\mathrm{pl}}$ is positively correlated to the $\mathrm{CX}$ ratio and negatively correlated to Lc. $\rho_{1}$ whereas $\mathrm{J}$ is negatively correlated to Lc. $\rho_{1}$ (Table S2).

In the radial diaphysis, no significant correlation was found for neither of the two loading conditions (Table S3).

We now consider the study over the 24 pooled samples (Table 1). Under quasi-static loading, $\mathrm{J}_{\mathrm{el}}$ and $\mathrm{J}_{\mathrm{pl}}$ as well as $\mathrm{J}$ were negatively correlated with Ca.Dm. All three toughness parameters $\mathrm{J}_{\mathrm{el}}, \mathrm{J}_{\mathrm{pl}}$, and $\mathrm{J}$ were positively correlated with Lc. $\rho_{1} . \mathrm{J}_{\mathrm{pl}}$, related to crack propagation, in particular showed a relatively strong correlation. Under fall-like loading conditions, $\mathrm{J}_{\mathrm{el}}$ is negatively correlated to Ca.Dm (Table 1). Table $\mathrm{S} 4$ (supplementary materials) presents similar results as Table 1 with more biochemical and structural parameters.

Table 1: Spearman's coefficient between the biomechanical, biochemical and structural parameters.

\begin{tabular}{|c|}
\hline Biomechanical \\
\hline
\end{tabular}

\begin{tabular}{c|ccccc}
\hline & QS & $-0.42^{*}$ & -0.27 & $-0.51^{*}$ & -0.18 \\
& $F$ & $-0.42^{*}$ & -0.19 & -0.17 & 0.21 \\
\hline \multirow{3}{*}{$J_{p l}$} & QS & $-0.45^{*}$ & -0.13 & $-0.62^{* *}$ & 0.05 \\
& $F$ & 0.04 & -0.09 & 0.04 & 0.31 \\
\hline & QS & $-0.45^{*}$ & -0.14 & $-0.66^{* *}$ & 0.06 \\
& $F$ & -0.07 & -0.15 & -0.13 & 0.28 \\
\hline
\end{tabular}

${ }^{*} p<0.05 ;{ }^{* *} p<0.01$ 
Table 2 presents the best multivariate linear regression explaining biomechanical variances with structural and biochemical parameters, even considering all the biochemical and structural parameters presented in Table S4.

Multiple linear regression revealed several significant relations. Under quasi-static loading, for the elastic and plastic contribution of the toughness, the combination of On.Ca.Dm, Lc.N/BV and Lc.p1 explains the variance in $\mathrm{J}_{\mathrm{el}}$ up to $32 \%$, and the combination of the CX ratio, On.Ca.Dm and Lc.pl explains the variance of $\mathrm{J}_{\mathrm{pl}}$ and $\mathrm{J}$ up to $50 \%$ and $51 \%$, respectively.

Under fall-like loading, the combination of On.Ca.Dm, the CX ratio and Lc.N/BV explains the variance in $\mathrm{J}_{\mathrm{el}}$ up to $39 \%$.

Table 2: Multivariate linear regression explaining the variance of biomechanical parameters.

\begin{tabular}{|c|c|c|c|c|}
\hline & & Best combination & p-value & Adj-R $R^{2}$ \\
\hline \multirow{2}{*}{$\mathrm{Jel}_{\mathrm{e}}$} & QS & $1.57-0.002 \times$ On. Ca. Dm $-2.16 .10^{-5} \times L c . \frac{N}{B V}-0.08 \times$ Lc. $\rho_{1}$ & 0.02 & 0.32 \\
\hline & $F$ & $2.49-0.01 \times$ On. Ca. $D m-7.68 .10^{-5} \times L c . \frac{N}{B V}+0.29 \times C X$ & 0.007 & 0.39 \\
\hline \multirow{2}{*}{$J_{\mathrm{pl}}$} & QS & $30.00-0.11 \times$ On. Ca. Dm - $2.90 \times$ Lc. $\rho_{1}+2.39 \times C X$ & 0.007 & 0.50 \\
\hline & $F$ & - & - & - \\
\hline \multirow{2}{*}{$J$} & QS & $31.11-0.11 \times$ On. Ca. $D m-2.99 \times$ Lc. $\rho_{1}+2.39 \times C X$ & 0.001 & 0.51 \\
\hline & $F$ & - & - & - \\
\hline
\end{tabular}

QS: quasi-static ; F: fall

Figure 3 shows the estimate of $\mathbf{J}$ using the best combinations shown in table 2 as a function of the real measure of $\mathrm{J}$. 


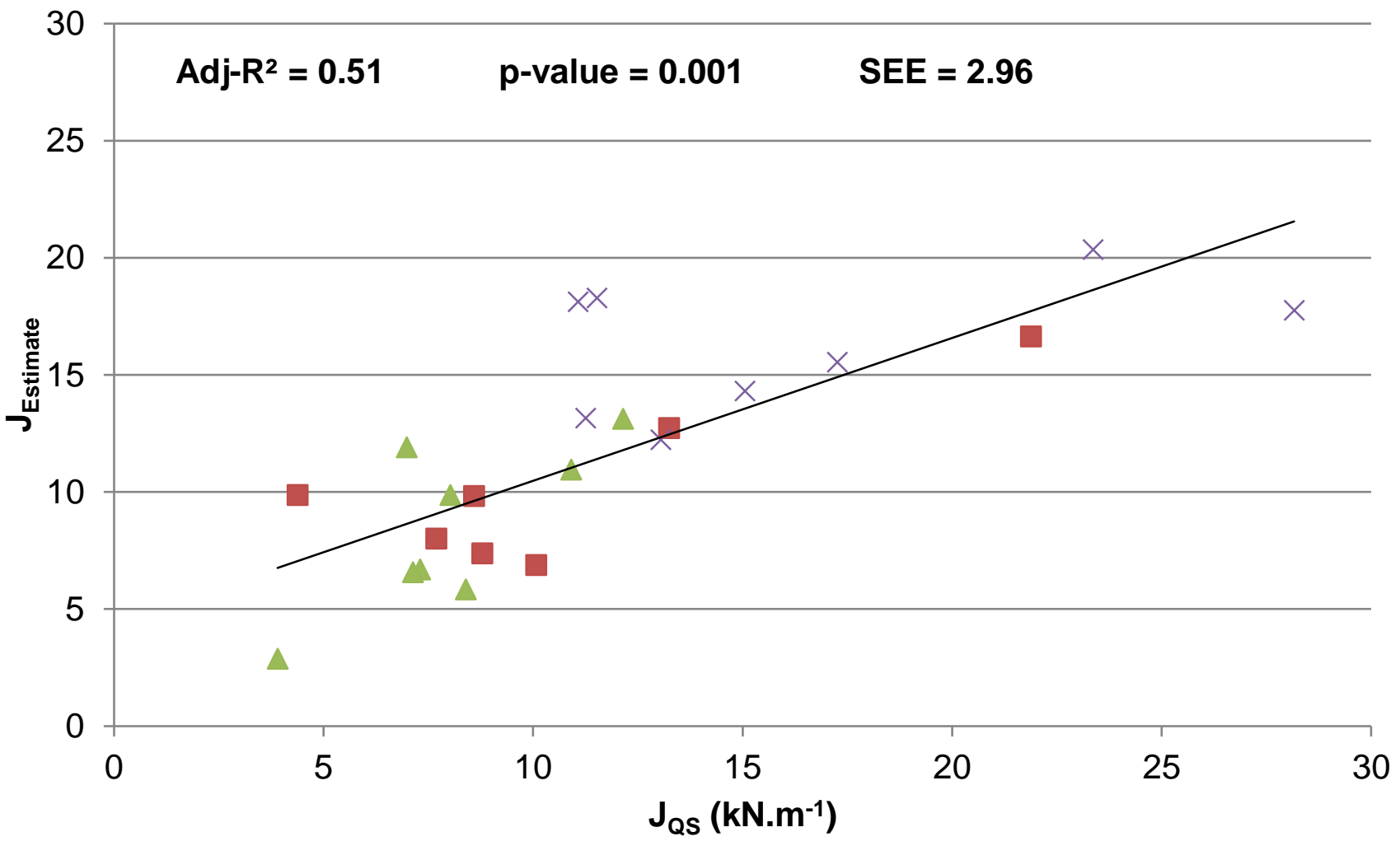

Femoral diaphysis $\quad \Delta$ Femoral neck $\quad \times$ Radial diaphysis

Figure 3: JQS measured in fucntion of $J_{E s t i m a t e}$ using the equation in Table 2 (SEE = Standard Error of the Estimate).

\section{Discussion}

A better knowledge of human cortical bone crack propagation mechanisms is of great interest for a better care of bone fracture risk. Cortical bone is a complex material showing a multi-scale arrangement of organic and mineral ${ }^{31}$. In this study performed on 8 paired human femoral diaphyses, femoral necks and radial diaphyses, we investigated the influence on human cortical bone toughness of the Haversian system, the lacunar system, and the collagen matrix maturation at two loading conditions. The use of different anatomical locations allows the investigation of crack propagation mechanisms over a wide range of mechanical or organizational properties. To our knowledge, this is the first study to investigate human cortical bone toughness involving structural and compositional features at different length scales and considering two loading conditions.

Considering the elastic regime, we can observe that both the canal diameter and lacunar radius of curvature are correlated with the elastic toughness under quasi-static loading. Significant correlations between the elastic toughness and the Haversian system have already been found in the past ${ }^{32-34}$. A significant decrease of the yield stress with the 
increase of the canal diameter has also been observed ${ }^{35}$. These results are in agreement with our results. The initiation of the crack is governed by the stretching of the tissue in the neighborhood of the notch, thus involving the stretching of mineralized collagen molecules ${ }^{36}$. It has been shown that the elastic behavior at the tissue level is associated with the behavior at the fibrillar or mineral scale ${ }^{37}$. It is also known that the Haversian canal parameters are correlated with bone elastic properties ${ }^{38-40}$, which is confirmed here as we find a significant correlation between canal diameter and elastic toughness. A relationship between the initiation toughness and the lacunar system has also previously been observed ${ }^{33}$. There is fewer data available at this scale, however.

The hypothesis is here that lacunae may play a role of stress concentrators ${ }^{41-44}$. It is known from the field of fracture mechanics that small defects have the capacity to concentrate stresses during the loading ${ }^{45,46}$. This means that the stress around these defects is higher than in the surrounding environment (see on Figure 1 the strain concentration around the notch). It has been shown that the strain in the neighborhood of lacunae was increased relative to the surrounding tissue ${ }^{41,43,47}$. By playing the role of such defects, the lacunae can concentrate some stresses during the loading, and thus relax the notch. Actually, for a same mechanical energy provided to the material, if the notch is placed in an homogeneous medium, all the energy will be concentrated around it ; nevertheless if other small defects surround this notch, some of the total energy will be used to concentrate the stress around these small defects, thus the stress concentration state around the notch will be lowered This will increase the energy needed to initiate a crack from the notch. Furthermore, a stress concentrator with a smaller radius of curvature will concentrate even more stress ${ }^{46}$, which supports the negative correlation we found here. Regarding the multiple linear regression, we can observe that the lacunar density help to better estimate the elastic toughness. This suggests that when cortical bone has a lower density of lacunae, it can better resist crack propagation. The hypothesis to explain this results is related to the previous one considering lacunae as stress concentrators. If the concentration of these stress concentrators is too high close to the notch, the stress concentration state of the sample near this notch may increase more rapidly because of a higher density of stress concentrators, decreasing thus the energy needed to initiate the crack.

Considering fall like loading, there is also here a significant correlation between canal diameter and elastic toughness. As stated previously, it may imply that the initiation toughness is associated with the elasticity of the tissue. However, there is no correlation between the elastic toughness and the lacunar radius of curvature at this higher strain rate. As the loading is applied faster, the stress field may expand faster, and there might not be enough time for stress to concentrate sufficiently around the lacunae to relax the notch. We were able to predict the initiation toughness in the case of fall-like loading with better accuracy than under quasi-static loading, based on the lacunar density, the CX ratio, and the canal diameter.

Further, we can observe that the non-linear part of the deformation and the global toughness ( $\mathrm{J}_{\mathrm{pl}}$ and $\mathrm{J}$, respectively) are both similarly correlated with structural and biochemical parameters (cf. Table 1 and Table 2). This is due to the fact that cortical bone crack propagation is mainly governed by these non-linear mechanisms, both under 
quasi-static and fall-like loading. Furthermore, these non-linear mechanisms are more affected by the increase of the strain rate than the linear mechanisms ${ }^{24,26}$. Therefore it is important to elucidate these mechanisms. From the results given in Table 1 we can observe that under quasi-static loading, the non-linear mechanisms of bone crack propagation are related to the canal diameter. Where the canals are larger, cracks have less difficulty to pass through the bone microstructure.

Relationships between the Haversian systems and the global toughness $\mathbf{J}$ have already been evidenced in a previous study ${ }^{33}$. It is well known that the osteons, which surround the vascular canals, play a major role in crack propagation mechanisms. When a crack encounters the interface between the interstitial tissue and the osteon, it can be arrested or deviated along this interface ${ }^{15,48,49}$. This role as a barrier to crack propagation may come from the difference of elasticity between the osteon and its surrounding tissue ${ }^{20,50}$. Illustration of these mechanisms can be seen in ${ }^{51}$, where $\mathrm{SR} \mu \mathrm{CT}$ was also used to image the samples. The hypothesis is thus that the correlation between bone toughness and the canal diameter is likely to be due to the influence of the osteon morphology on crack propagation, the morphology of the osteons being dependent on the on morphology of the Haversian canals ${ }^{52}$. Furthermore, a previous study has shown that the pullout of the osteon from the interstitial tissue required less energy for larger osteons ${ }^{53}$. The authors hypothesized that for larger canals, the shear stress at the cement line is larger relative to the tensile stress applied to the whole osteon, resulting in an easier debonding at the interface. This result is in accordance with the present study that state that cracks propagate more easily in a microstructure with larger canals.

We can also observe a significant relationship between the non-linear toughness and the radius of curvature of the lacunae. To the authors' knowledge, there is no data about this in the literature. It is however known that the lacunae can guide the cracks during propagation 47,54 . This is in accordance with the previously mentioned hypothesis that osteocyte lacunae act as stress concentrators. Christen et al. also hypothesized that the stress concentration near the lacunae may involve a blunting of the crack, resulting in increased toughness ${ }^{47}$. This supports the results found here and highlights the stress concentrators, and thus energy dissipating, role played by osteocyte lacunae during crack propagation under quasi-static loading.

Finally we found that the CX ratio improves the estimation of the toughness, both the plastic and the global, in a multi variate model. We have shown in a previous study that the maturation of the enzymatic cross-links may have a minor influence on human cortical bone toughness ${ }^{26}$. The present results support this minor effect. The associated mechanisms may be the interfibrillar sliding that consumes energy during loading ${ }^{12,55}$. With a higher CX ratio, the tissue contains more immature cross-links relative to mature cross-links. As these mature cross-links are divalent compared to the trivalent mature cross-links, they may need less energy to break and thus encourage this interfibrillar sliding mechanism.

Under fall-like loading, we found no significant correlation between the non-linear toughness and structural or compositional features. This suggests that in the case of a fall, the mechanisms described above are much less efficient. Because of the higher loading 
rate, the microstructure's response to crack propagation may not be the same, for example because of the time dependent nature of some bone features. It is known that at higher loading rate, there is a stiffening of bone tissue ${ }^{56}$. In a previous study, based on the "He and Hutchinson framework", Zimmerman et al. hypothesized that the stiffening of the tissue may change the osteons deflection capacity ${ }^{20,50}$. This stiffening of the tissue may also modify the strain concentration effect around the osteocyte lacunae, resulting in a straighter crack propagation path.

Regarding the correlations results, the linear regressions (Table 1) obtained in the current study may appear quite low compare to other studies investigating the relationships between cortical bone biomechanical properties and biochemical or structural properties ( $\rho$ Spearman $=0.66$ in the current study, $r=0.78$ in ${ }^{57}, r=0.73$ in ${ }^{58}, r=0.94$ in ${ }^{59}$ ). In most of the studies, the biomechanical parameter investigated was bone strength (MPa) using continuum mechanics, whereas in the current study the investigated parameters is the toughness $\left(\mathrm{MPa}^{\mathrm{m}} \mathrm{H}^{0.5}\right.$ ) and more particularly the associated strain energy release rate ${ }^{24}$, are related to the fracture mechanics field. Strength and toughness are two mechanical parameters that provide information on the ultimate mechanical properties of the studied materials. Nevertheless, they do not give the same information and it has been shown that bone strength is poorly correlated to the global toughness $(\mathrm{J}){ }^{60}$. Strength is the mechanical stress at which the deformation of the material becomes non-recoverable ${ }^{61}$. The toughness of bone tissue gives information on the mechanical energy that is needed to initiate and propagate a crack from a pre-existing defect $45,46,62,63$. ). This results in a parameter that is dependent on a local area surrounding the notch. Some previous works already studied the correlation coefficient between bone toughness and bone parameters. For instance, a low $\mathrm{R}^{2}$ between bone toughness and the degree of mineralisation has been measured for the elastic toughness $\left(\rho_{\text {Spearman }}=0.29\right.$ in $\left.^{64}\right)$ and as non-significant when dealing with the global toughness ${ }^{33,64}$ whereas the correlation is significant between bone strength and mineralisation ${ }^{57-59}$. The toughness of bone might be more sensitive to the structure heterogeneities observable in cortical bone tissue ${ }^{65,66}$. This is why the authors investigated the relationships between bone toughness and bone cortical structure at a lower length scale, such as the lacunar scale.

The main limitations of this study are related to the population investigated. The population used for the assessment of bone mechanical and structural properties comprised only 8 donors, which is a relatively small effective population. The main reasons for the limited sample size is the limited access to image acquisition time at synchrotron facilities, and the very large data sets that this modality yields. Despite of this, it can be seen that the population offers a large variety of mechanical, structural and compositional properties ${ }^{24-26}$. This somewhat alleviates the potential problem of a small population size. The wide range of properties still allowed the differences in structural and compositional properties to explain the differences in mechanical properties. Further, the population is not representative of the general population, in terms of gender and age. We investigated a population of aged female (50 y.o. to 91 y.o.) donors. It is known, however, that the population of post-menopausal women is more at risk of bone fracture 67,68 . It is thus relevant to assess bone fracture properties on such a population.

The results obtained here show that, in order to improve our biomechanical knowledge on bone fracture, it is relevant to investigate bone mechanical properties at rates that are 
representative of the reality of falls. It is clear that bone fracture mechanisms are highly dependent on the loading rate. While these mechanisms are widely studied and well known under standard, quasi-static loading, there is still a substantial lack of data regarding higher loading rates. Therefore, the development of mechanical models of bone under realistic solicitation due to a fall, both considering loading rate and loading direction, represents a real challenge and will be highly useful for the improvement of prediction and prevention of bone fracture.

\section{Conclusion}

In this study, we have investigated the relationships between cortical bone toughness and its structural and compositional properties considering two loading conditions, representing walking and falls. Significant influence of the structure and composition on the initiation toughness was found in the two loading conditions. However, while the propagation of the crack is influenced by microstructural and compositional features under quasi-static loading, these features have less importance in the case of a fall. Further, since non-linear crack propagation mechanisms account for a large part of the global toughness, it will be important to study why an increase of the loading rate has a drastic effect on them.

\section{Acknowledgements}

The authors want to thank Cécile Olivier, Evelyne Gineyts, Leila Benboubaker and Pierre-Jean Gouttenoire for their technical support. The authors acknowledge the ESRF ID19 staff for its support for synchrotron data acquisition during the experiment MD923. This work was supported by the LabEx PRIMES (ANR-11-LABX-0063) within the program "Investissements d'Avenir" (ANR-11-IDEX-0007). This study was also partly funded by the Région Auvergne-Rhône-Alpes (14-011125-01) and by the ANR project MULTIPS (ANR-13-BS09-0006).

\section{References}

1. Siris ES, Chen Y-T, Abbott T a, et al. Bone mineral density thresholds for pharmacological intervention to prevent fractures. Arch Intern Med 2004; 164 : 1108-1112.

2. Siris ES, Adler R, Bilezikian J, et al. The clinical diagnosis of osteoporosis: A position statement from the National Bone Health Alliance Working Group. Osteoporos Int 2014; 25: 1439-1443.

3. Johnell O, Kanis J a. An estimate of the worldwide prevalence, mortality and disability associated with hip fracture. Osteoporos Int 2006; 15: 897-902.

4. Genant HK, Engelke K, Fuerst T, et al. Noninvasive assessment of bone mineral and structure: state of the art. J Bone Miner Res 1996; 11: 707-730.

5. Wachter NJ, Augat P, Krischak GD, et al. Prediction of cortical bone porosity in vitro by microcomputed tomography. Calcif Tissue Int 2001; 68: 38-42.

6. van Rietbergen B, Ito K. A survey of micro-finite element analysis for clinical assessment of bone strength: The first decade. J Biomech 2015; 48: 832-841. 
7. Seeman E, Delmas PD. Bone quality--the material and structural basis of bone strength and fragility. N Engl J Med 2006; 354: 2250-2261.

8. Yeni YN, Brown CU, Gruen T a., et al. The relationships between femoral cortex geometry and tissue mechanical properties. J Mech Behav Biomed Mater 2013; 21: 9-16.

9. Ritchie RO. Mechanisms of fatigue crack propagation in metals, ceramics and composites: Role of crack tip shielding. Mater Sci Eng A 1988; 103: 15-28.

10. Nalla RK, Kruzic JJ, Ritchie RO. On the origin of the toughness of mineralized tissue: Microcracking or crack bridging? Bone 2004; 34: 790-798.

11. Zimmermann EA, Schaible E, Bale H, et al. Age-related changes in the plasticity and toughness of human cortical bone at multiple length scales. Proc Natl Acad Sci 2011; 108: 14416-14421.

12. Gupta HS, Krauss S, Kerschnitzki M, et al. Intrafibrillar plasticity through mineral/collagen sliding is the dominant mechanism for the extreme toughness of antler bone. J Mech Behav Biomed Mater 2013; 28: 366-382.

13. Tang SY, Vashishth D. Non-enzymatic glycation alters microdamage formation in human cancellous bone. Bone 2010; 46: 148-154.

14. Zioupos P, Hansen U, Currey JD. Microcracking damage and the fracture process in relation to strain rate in human cortical bone tensile failure. J Biomech 2008; 41: 2932-2939.

15. O'Brien FJ, Taylor D, Lee TC. The effect of bone microstructure on the initiaton and growth of microcracks. J Orthop Res 2005; 23: 475-480.

16. Burr DB, Schaffler MB, Frederickson RG. Composition of the cement line and its possible mechanical role as a local interface in human compact bone. J Biomech 1988; 21: 939-945.

17. Koester KJ, Ager JW, Ritchie RO. The true toughness of human cortical bone measured with realistically short cracks. Nat Mater 2008; 7: 672-677.

18. Berteau J-P, Gineyts E, Pithioux M, et al. Ratio between mature and immature enzymatic cross-links correlates with post-yield cortical bone behavior: An insight into greenstick fractures of the child fibula. Bone 2015; 79: 190-195.

19. Depalle B, Qin Z, Shefelbine SJ, et al. Influence of cross-link structure, density and mechanical properties in the mesoscale deformation mechanisms of collagen fibrils. J Mech Behav Biomed Mater 2015; 52: 1-13.

20. Zimmermann EA, Gludovatz B, Schaible E, et al. Fracture resistance of human cortical bone across multiple length-scales at physiological strain rates.

Biomaterials 2014; 35: 5472-5481.

21. Ural A, Zioupos P, Buchanan D, et al. The effect of strain rate on fracture toughness of human cortical bone: A finite element study. J Mech Behav Biomed Mater 2011; 4: 1021-1032.

22. Kulin RM, Jiang F, Vecchio KS. Loading rate effects on the R-curve behavior of cortical bone. Acta Biomater 2011; 7: 724-732.

23. Tinetti ME. Preventing falls in elderly persons. N Engl J Med 2003; 348: 42-49.

24. Gauthier R, Follet H, Langer M, et al. Strain rate influence on human cortical bone toughness: A comparative study of four paired anatomical sites. J Mech Behav Biomed Mater 2017; 71: 223-230.

25. Gauthier R, Langer M, Follet H, et al. 3D micro structural analysis of human 
cortical bone in paired femoral diaphysis, femoral neck and radial diaphysis. $J$ Struct Biol 2018; 204: 182-190.

26. Gauthier R, Follet H, Langer M, et al. Relationships between human cortical bone toughness and collagen cross-links on paired anatomical locations. Bone 2018; 112: 202-211.

27. Foldhazy Z. Exercise-induced strain and strain rate in the distal radius. $J$ Bone $J t$ Surg - Br Vol 2005; 87-B: 261-266.

28. American Society for Testing and Materials. Standard Test Method for LinearElastic Plane-Strain Fracture Toughness of Metallic Materials. Annu B ASTM Stand 1997; 03: E 399 - 90.

29. Irwin GR. Analysis of stresses and strains near the end of cracking traversing a plate. J Appl Mech 1957; 24: 361-364.

30. Dong P, Haupert S, Hesse B, et al. 3D osteocyte lacunar morphometric properties and distributions in human femoral cortical bone using synchrotron radiation micro-CT images. Bone 2014; 60: 172-185.

31. Rho JY, Kuhn-Spearing L, Zioupos P. Mechanical properties and the hierarchical structure of bone. Med Eng Phys 1998; 20: 92-102.

32. Yeni YN, Brown CU, Wang Z, et al. The influence of bone morphology on fracture toughness of the human femur and tibia. Bone 1997; 21: 453-459.

33. Granke M, Makowski AJ, Uppuganti S, et al. Prevalent role of porosity and osteonal area over mineralization heterogeneity in the fracture toughness of human cortical bone. J Biomech 2016; 49: 2748-2755.

34. Tang SY, Vashishth D. The relative contributions of non-enzymatic glycation and cortical porosity on the fracture toughness of aging bone. J Biomech 2011; 44: 330-336.

35. Wachter N., Krischak G., Mentzel M, et al. Correlation of bone mineral density with strength and microstructural parameters of cortical bone in vitro. Bone 2002; 31: 90-95.

36. Nair AK, Gautieri A, Chang S-W, et al. Molecular mechanics of mineralized collagen fibrils in bone. Nat Commun 2013; 4: 1724.

37. Gupta HS, Seto J, Wagermaier W, et al. Cooperative deformation of mineral and collagen in bone at the nanoscale. Proc Natl Acad Sci U S A 2006; 103: 1774117746.

38. Granke M, Grimal Q, Saïed A, et al. Change in porosity is the major determinant of the variation of cortical bone elasticity at the millimeter scale in aged women. Bone 2011; 49: 1020-1026.

39. Parnell WJ, Grimal Q. The influence of mesoscale porosity on cortical bone anisotropy. Investigations via asymptotic homogenization. J R Soc Interface 2009; 6: 97-109.

40. Bala Y, Lefevre E, Roux JP, et al. Pore network microarchitecture influences human cortical bone elasticity during growth and aging. J Mech Behav Biomed Mater 2016; 63: 164-173.

41. Rath Bonivtch A, Bonewald LF, Nicolella DP. Tissue strain amplification at the osteocyte lacuna: A microstructural finite element analysis. J Biomech 2007; 40: 2199-2206.

42. Verbruggen SW, Vaughan TJ, McNamara LM. Strain amplification in bone 
mechanobiology: a computational investigation of the in vivo mechanics of osteocytes. J R Soc Interface 2012; 9: 2735-2744.

43. Varga P, Hesse B, Langer M, et al. Synchrotron X-ray phase nano-tomographybased analysis of the lacunar-canalicular network morphology and its relation to the strains experienced by osteocytes in situ as predicted by case-specific finite element analysis. Biomech Model Mechanobiol. Epub ahead of print 2014. DOI: 10.1007/s10237-014-0601-9.

44. Nicolella DP, Moravits DE, Gale AM, et al. Osteocyte lacunae tissue strain in cortical bone. J Biomech 2006; 39: 1735-1743.

45. Griffith a. a. The Phenomena of Rupture and Flow in Solids. Philos Trans R Soc A Math Phys Eng Sci 1921; 221: 163-198.

46. Inglis CE. Stresses in a plate due to the presnce of cracks and sharp corners. Trans. I.N.A. 1913; XLIV: 15.

47. Christen D, Levchuk A, Schori S, et al. Deformable image registration and 3D strain mapping for the quantitative assessment of cortical bone microdamage. $J$ Mech Behav Biomed Mater 2012; 8: 184-193.

48. Yeni YN, Norman TL. Calculation of porosity and osteonal cement line effects on the effective fracture toughness of cortical bone in longitudinal crack growth. $J$ Biomed Mater Res 2000; 51: 504-509.

49. Mischinski S, Ural A. Interaction of microstructure and microcrack growth in cortical bone: a finite element study. Comput Methods Biomech Biomed Engin 2011; 1-14.

50. He M-Y, Hutchinson JW. Kinking of a Crack Out of an Interface. J Appl Mech 1989; 56: 270.

51. Wolfram U, Schwiedrzik JJ, Mirzaali MJ, et al. Characterizing microcrack orientation distribution functions in osteonal bone samples. J Microsc 2016; 00: 114.

52. Qiu S, Fyhrie DP, Palnitkar S, et al. Histomorphometric assessment of Haversian canal and osteocyte lacunae in different-sized osteons in human rib. Anat Rec Part A Discov Mol Cell Evol Biol 2003; 272: 520-525.

53. van Oers RFM, Ruimerman R, van Rietbergen B, et al. Relating osteon diameter to strain. Bone 2008; 43: 476-482.

54. Voide R, Schneider P, Stauber M, et al. Time-lapsed assessment of microcrack initiation and propagation in murine cortical bone at submicrometer resolution. Bone 2009; 45: 164-173.

55. Hansma PK, Fantner GE, Kindt JH, et al. Sacrificial bonds in the interfibrillar matrix of bone. J Musculoskelet Neuronal Interact 2005; 5: 313-315.

56. Hansen U, Zioupos P, Simpson R, et al. The effect of strain rate on the mechanical properties of human cortical bone. J Biomech Eng 2008; 130: 011011.

57. Louis O, Boulpaep F, Willnecker J, et al. Cortical mineral content of the radius assessed by peripheral QCT predicts compressive strength on biomechanical testing. Bone 1995; 16: 375-379.

58. Hsu JT, Wang SP, Huang HL, et al. The assessment of trabecular bone parameters and cortical bone strength: A comparison of micro-CT and dental cone-beam CT. $J$ Biomech 2013; 46: 2611-2618.

59. Zysset PK, Dall'ara E, Varga P, et al. Finite element analysis for prediction of 
bone strength. Bonekey Rep 2013; 2: 386.

60. Zioupos P, Kaffy C, Currey JD. Tissue heterogeneity, composite architecture and fractal dimension effects in the fracture of ageing human bone. Int J Fract 2006; 139: 407-424.

61. Ritchie RO. The conflicts between strength and toughness. Nat Mater 2011; 10: 817-822.

62. Rice JR, Rosengren GF. Plane strain deformation near a crack tip in a power-law hardening material. J Mech Phys Solids 1968; 16: 1-12.

63. Nalla RK, Stölken JS, Kinney JH, et al. Fracture in human cortical bone: Local fracture criteria and toughening mechanisms. J Biomech 2005; 38: 1517-1525.

64. Granke M, Makowski AJ, Uppuganti S, et al. Identifying Novel Clinical Surrogates to Assess Human Bone Fracture Toughness. J Bone Miner Res 2015; 30: $1290-1300$.

65. Zimmermann EA, Ritchie RO. Bone as a Structural Material. Adv Healthc Mater 2015; 4: 1287-1304.

66. McCormack J, Stover SM, Gibeling JC, et al. Effects of mineral content on the fracture properties of equine cortical bone in double-notched beams. Bone 2012; 50: $1275-1280$.

67. Kimmel DB, Recker RR, Gallagher JC, et al. A comparison of iliac bone histomorphometric data in post-menopausal osteoporotic and normal subjects. Bone Miner 1990; 11: 217-235.

68. Court-Brown CM, Caesar B. Epidemiology of adult fractures: A review. Injury 2006; 37: 691-697. 